\title{
The loss of the BH3-only Bcl-2 family member Bid delays T-cell leukemogenesis in Atm - I- mice
}

\author{
S Biswas ${ }^{1,2}, \mathrm{Q} \mathrm{Shi}^{1}$, A Wernick${ }^{1}$, A Aiello ${ }^{1}$ and SS Zinkel ${ }^{* 1}$
}

Multicellular organisms maintain genomic integrity and resist tumorigenesis through a tightly regulated DNA damage response (DDR) that prevents propagation of deleterious mutations either through DNA repair or programmed cell death. An impaired DDR leads to tumorigenesis that is accelerated when programmed cell death is prevented. Loss of the ATM (ataxia telangiectasia mutated)-mediated DDR in mice results in T-cell leukemia driven by accumulation of DNA damage accrued during normal T-cell development. Pro-apoptotic BH3-only Bid is a substrate of Atm, and Bid phosphorylation is required for proper cell cycle checkpoint control and regulation of hematopoietic function. In this report, we demonstrate that, surprisingly, loss of Bid increases the latency of leukemogenesis in Atm - I - mice. Bid - I - Atm - I - mice display impaired checkpoint control and increased cell death of DN3 thymocytes. Loss of Bid thus inhibits T-cell tumorigenesis by increasing clearance of damaged cells, and preventing propagation of deleterious mutations.

Cell Death and Differentiation (2013) 20, 869-877; doi:10.1038/cdd.2013.16; published online 8 March 2013

Cell survival depends on careful maintenance of genomic integrity. Cells manage DNA damage by activating cell cycle checkpoints and DNA repair, or programmed cell death if the damage is too extensive. ${ }^{1,2}$ Failure to sense DNA damage causes increased incidence of cancer in mice and humans bearing mutations of genes involved in DNA damage response (DDR) pathways. ${ }^{3}$

Two kinases, Atm and Atr, mediate the cellular response to DNA damage. ${ }^{3,4}$ These kinases sense DNA damage, and activate downstream effectors to initiate cell cycle checkpoints and DNA repair, or cell death. Atm is activated primarily in response to double-strand DNA breaks and oxidative stress. ${ }^{5-7}$ Atr is activated by single strand DNA, such as those that accumulates during polymerase stalling during replicative stress. ${ }^{8-10}$ There is significant overlap in the Atm and Atr signaling pathways and activation signals. ${ }^{11}$ In addition, loss of function of one kinase results in compensatory upregulation of the other. ${ }^{12}$ For example, cells deficient in Atm display increased Atr activity that can partially compensate to monitor and repair DNA damage. ${ }^{12}$

The DDR is closely linked to pathways activating programmed cell death. Pro-apoptotic Bax is transcriptionally upregulated by p53 following DNA damage. ${ }^{13}$ PUMA, a member of the BH3-only subgroup of the Bcl-2 family, activates Bax and Bak following ionizing radiation (IR). ${ }^{14,15}$

Several independent groups have shown that $\mathrm{BH}$-only Bid has a role in the DDR independent of it pro-apoptotic role. ${ }^{16-18}$ Bid-/ - myeloid progenitor cells demonstrate increased sensitivity to agents inducing replicative stress. Bid is phosphorylated by the DNA damage kinases, Atm and/or
Atr, and mediates the intra-S phase checkpoint. ${ }^{17}$ Furthermore, Bid associates with members of the DNA damage sensor complex, Atrip, the obligate Atr-binding partner, and Replication protein $A$, and mediates the formation and/or stability of the DNA damage sensor complex. ${ }^{19}$ Finally, loss of Bid results in an impaired ability of hematopoietic stem cells (HSC) and progenitor cells to respond to chronic replicative stress induced by hydroxyurea (HU). ${ }^{20}$ Mice challenged with repeated doses of $\mathrm{HU}$ display decreased $\mathrm{HSC}$ and progenitor cells' function. Bid thus functions at the level of the DNA damage sensor complex, and integrates DNA replicative stress signals. ${ }^{19-21} A$ recent report, using mice harboring Bid mutated in the Atm/Atr phosphorylation sites that has been knocked into the Bid locus, has demonstrated that Atmmediated Bid phosphorylation has a role in protecting HSCs from irradiation and oxidative stress, suggesting the possibility that Bid may have a more general role in sensing stress. ${ }^{22}$

An elegant series of experiments has recently demonstrated in the mouse model of lymphomagenesis induced by $\gamma$ irradiation that tumorigenesis is driven by repeated cycles of hematopoietic progenitor cell death, inducing compensatory mobilization of HSC and progenitor cells. ${ }^{23}$ This proliferation results in increased accumulation of mutations in the vulnerable HSC and progenitor cell populations. Loss of Noxa, ${ }^{23,24}$ (as well as $\mathrm{Bad}$ and $\mathrm{Bim}^{24}$ ) accelerates lymphomagenesis. Interestingly, Puma loss ablates IR-induced lymphomagenesis by specifically preventing cell death of hematopoietic progenitors, thus preventing the HSC mobilization that promotes acquisition of oncogenic mutations. These studies demonstrate the

\footnotetext{
${ }^{1}$ Department of Medicine, Vanderbilt University Medical Center, Nashville, TN, USA

*Corresponding author: SS Zinkel, Department of Medicine, Vanderbilt University Medical Center, 2220 Pierce Avenue, 548 PRB, Nashville, TN 37232 , USA. Tel: + 1615936 1801; Fax: + 1615936 3853; E-mail: Sandra.zinkel@vanderbilt.edu

${ }^{2}$ Current address: Institute of Liver and Biliary Sciences, New Delhi, India.

Keywords: apoptosis; DNA damage; leukemia

Abbreviations: Atm, Ataxia telangiectasia mutated; Atr, Atm and Rad3-related; Atrip, Atr-interacting protein; Bid, BH3-interacting death domain agonist; BrdU, bromodeoxyuridine; CD, cluster of differentiation; CMML, chronic myelomonocytic leukemia; DCFDA, 2',7' dichlorofluorescein diacetate; DDR, DNA damage response; DN, double negative; DNA, deoxyribonucleic acid; DSBs, double-strand breaks; EPO, erythropoietin; HSC, hematopoietic stem cell; HU, hydroxyurea; IL-3, interleukin 3; IL-6, interleukin 6; IR, ionizing radiation; MPCs, myeloid progenitor cells; PIKKs, phosphoinositide 3-kinase related protein kinases; PUMA, p53 up-regulated modulator of apoptosis; ROS, reactive oxygen species; SCF, stem cell factor; TCR, T cell receptor

Received 23.7.12; revised 17.12.12; accepted 07.1.13; Edited by JM Hardwick; published online 08.3.13
} 
importance of hematopoietic homeostatic regulation on tumor initiation and progression, and clearly demonstrate that the unique properties of various $\mathrm{BH} 3$-only proteins in a given system influence the final biological outcome.

To further define the role of Bid in the DDR in vivo and dissect Bid's role in tumorigenesis in a tumor model induced by DNA damage, we crossed Bid - / - mice with Atm - Imice. Atm - / - mice develop T-cell lymphoblastic leukemia/ lymphoma with an average latency of 3 months, driven by impaired DNA repair during T cell receptor (TCR) rearrangement. TCR $\beta$-chain rearrangement occurs during the double negative 3 (DN3) stage of development, is mediated by Rag recombinase, and activates a DDR. Loss of the Rag recombinase abrogates leukemia development in Atm - Imice. ${ }^{25}$ We reasoned that if Bid has a pro-apoptotic role in the Atm-directed DDR, loss of Bid in addition to Atm might accelerate leukemogenesis by allowing propagation of oncogenic mutations. If Bid functions solely as an Atm effector, Bid-I-Atm-I- mice should phenocopy Atm-I- mice. As the Atr-mediated DDR is increased to compensate for the loss of Atm, loss of Bid in addition to Atm would be expected to further impair the compensatory Atr-mediated DDR, increasing the DNA damage accrued during thymocyte differentiation.

Surprisingly, Bid-I-Atm-I - mice develop T-cell lymphoblastic leukemia/lymphoma with an increased latency relative to Atm-I- mice (Figure 1a). Bid-I-Atm-Ithymi display increased number of immature DN3 thymocytes. These Bid-/-Atm-I- DN3 thymocytes display increased BrdU incorporation, increased annexin $\mathrm{V}$ positivity, and increased 53bp1 staining. These results are consistent with the reported effect of Bid on the DNA damage-induced intra-S phase checkpoint. ${ }^{17,18}$ This impaired checkpoint results in a failure to arrest DNA replication in the presence of DNA damage, resulting in increased cell death during the DN3 stage. We further show that Bid-I-Atm-Ithymocytes demonstrate decreased Chk1 phosphorylation, consistent with an impaired DDR. In addition, Bid-/Atm - / - thymocytes demonstrate increased tail moment in comet assays, and increased 53bp1 accumulation at foci, consistent with increased DNA damage. We thus propose a model in which loss of Bid further impairs the ability of DN3 thymocytes to carry out an effective DDR. This loss of a DDR delays tumorigenesis by increasing cell death.

\section{Results}

Loss of Bid attenuates leukemia development in Atm - I- mice. Bid is a substrate of Atm, phosphorylated at serines 61,64 , and 78 , following DNA damage. In addition, a recent report proposed that Bid serves as the dominant Atm substrate to mediate the response to oxidative stress in hematopoiesis. $^{22} \mathrm{Atm}-1-$ mice display impaired growth neurologic dysfunction, and male and female infertility secondary to the absence of mature gametes. ${ }^{26}$ In addition, Atm-I- mice develop T-cell lymphoblastic leukemia/ lymphoma with a latency of $\sim 3$ months.

To determine whether Bid is the dominant Atm effector in mediating the phenotypes of Atm-I- mice, we bred Bid-I- mice to Atm-I- mice. Surprisingly, although Bid - / - Atm - / - mice develop T-cell leukemia, the latency of leukemogenesis was significantly delayed to 190 days (Figure 1a), relative to the 90-day latency of leukemogenesis in Atm-I- mice. The $\mathrm{T}$ cell leukemia/lymphoma that develops in Bid - I-Atm - I - mice is indistinguishable from that of Atm - I - mice with respect to morphology of leukemic
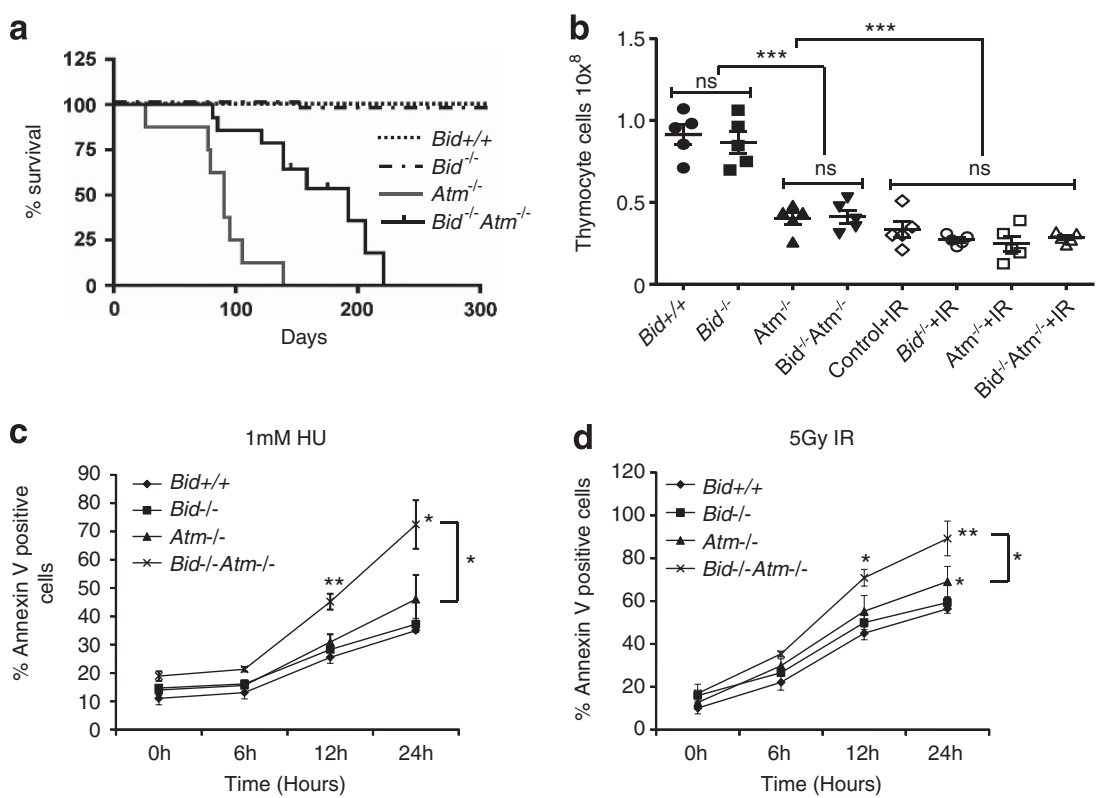

Figure 1 Loss of Bid attenuates T-cell lymphoblastic leukemia/lymphoma development in Atm $-/-$ mice. (a) Kaplan-Meier survival curves for cohorts of Bid $+/+$, Bid - I - , Atm - I - , and Bid - / - Atm - / - mice. (b) The total cellularity of control, Bid $+/+$, Bid $-/-$, Atm $-/-$, and Bid $-/-$ Atm $-/-$ thymocytes $48 \mathrm{~h}$ after 5 Gy IR treatment. (c, d). Thymocytes from Bid + /+, Bid - / - , Atm - / - , and Bid - I-Atm - / - mice were treated with (c) 1 mM hydroxyurea, or (d) 5 Gy ionizing radiation. Cell death was measured by annexin $\mathrm{V}$ staining with propidium iodine exclusion followed by flow cytometry. Data are representative of three independent experiments. ${ }^{*} P<0.05,{ }^{* *} P<0.01$ and ${ }^{* *} P<0.001$ 
blasts, size of the thymic mass, involvement of the thymus, spleen, and bone marrow, and immunophenotype of leukemic blasts (Supplementary Figure S2). In contrast, we found that loss of Bid does not rescue Atm - / - weight, thymus or spleen size, splenic $\mathrm{T}$ cells or defective spermatogenesis (Supplementary Figure S1 panels A-I, Figure 1b).

Bid-I-Atm-I- thymocytes are more sensitive to DNA damage. Atm-/- leukemogenesis is driven by ineffective DNA damage repair during T-cell development. To determine whether loss of Bid alters the response of thymocytes to DNA damage, we evaluated the response of thymocytes to IR that will induce predominantly an Atmdirected DDR, and $\mathrm{HU}$ that will induce predominantly an Atr-directed DDR. We irradiated mice with $5 \mathrm{~Gy} I \mathrm{R}$, and evaluated the thymocyte number after $48 \mathrm{~h}$. We found a similar number of thymocytes in Bid $+/+, B i d-/-$, Atm-/-, and Bid-/-Atm-/- mice following 5Gy IR. Compared to unirradiated mice, there is a significant decrease in thymus size following $\mathrm{IR}$ in $\mathrm{Bid}+/+$ and Bid-I - mice, but not in Atm-I- or Bid-I-Atm-Imice. Thus, loss of Bid does not alter the sensitivity of thymocytes to IR when administered to the whole animal (Figure 1b), consistent with the dispensable role of Bid in IR-mediated cell death. We next asked whether Bid mediates cell death in response to $\mathrm{HU}$ or IR in the absence of Atm. Interestingly, Bid-I-Atm-I- thymocytes display increased cell death following $1 \mathrm{mM} \mathrm{HU}$ or 5 Gy IR (Figures $1 \mathrm{c}$ and $\mathrm{d}$ ), suggesting that Bid may have a protective role in thymocytes in the absence of Atm.
Bid-I - Atm - I - mice accumulate DN3 thymocytes. In thymopoiesis, the population most vulnerable to the loss of a DDR is the DN3 population, as it is these cells that undergo TCR $\beta$ rearrangement. $R a g-/-A t m-/-$ mice do not develop leukemia, underscoring the importance of recombination to Atm - / - leukemogenesis. ${ }^{25}$

Bid has been reported to be important for cell death in DN3 thymocytes. $^{27}$ Bid transcription is stimulated by p53 in DN3 cells. Loss of Bid leads to the accumulation of DN3 cells in preTCR - / - mice, but does not result in further progenitor differentiation. $^{27}$ In addition, bone marrow reconstitution experiments with $\mathrm{Bid}-\mathrm{I}-$ bone marrow demonstrate decreased thymus size, but relative accumulation of DN3 thymocytes, further implicating Bid in regulation of DN3 thymocytes. ${ }^{28}$ As DN3 thymocytes are known to have a key role in T-cell leukemogenesis in the absence of Atm, and Bid is known to have a role in DN3 thymocytes, we evaluated DN3 thymocytes in Bid - I - Atm - I - mice. Loss of Atm leads to depletion of DN3 thymocytes. Surprisingly, Bid - I - Atm - I mice accumulate DN3 thymocytes above wild-type levels (Figures $2 \mathrm{a}$ and $\mathrm{b}$ ). The normal numbers of DN3 thymocytes in Bid-I - mice suggests that these results cannot be explained solely by loss of apoptosis in the absence of Bid.

In addition to its role in apoptosis, Bid mediates the intra-S phase checkpoint. ${ }^{17} \mathrm{Bid}-/-$ cells display aberrant radioresistant DNA synthesis, indicating a defective intra-S phase checkpoint following DNA damage. We therefore evaluated the proliferation of Bid-I - Atm - I - DN3 thymocytes. Bid-IAtm - / - thymocytes display increased proliferation relative to $\mathrm{Bid}+/+$, Bid $-/-$, or Atm - / - thymocytes (Figures $2 \mathrm{c}$ and a

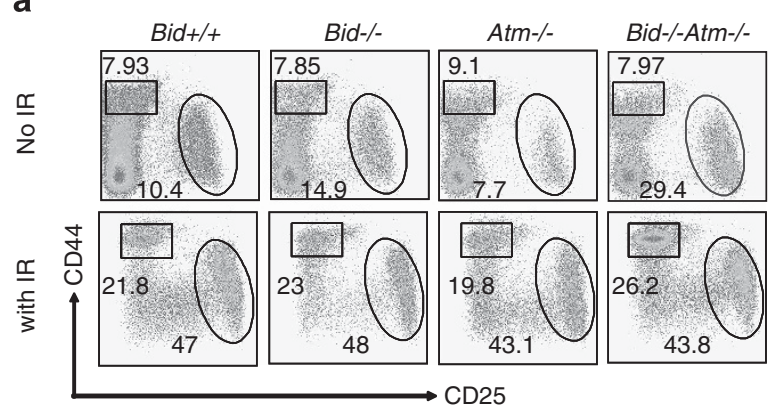

C

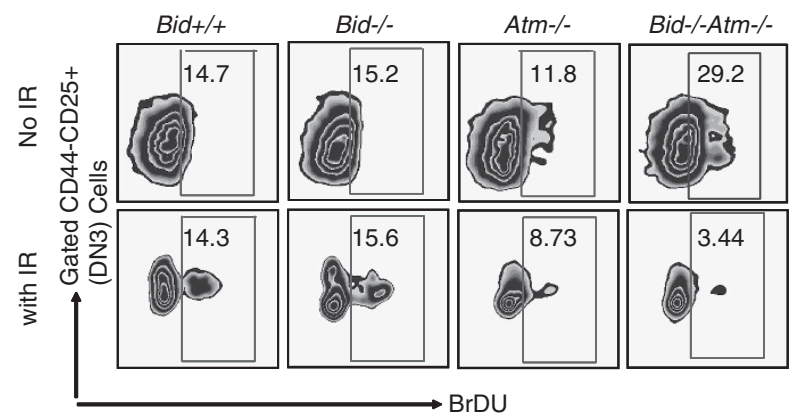

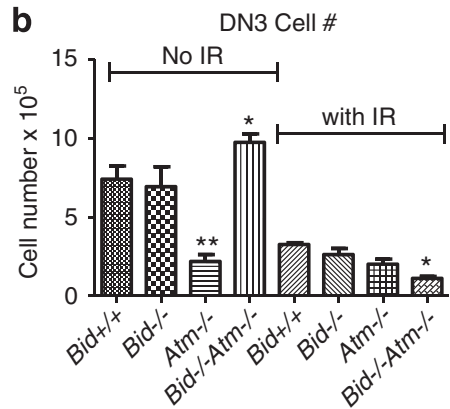

d

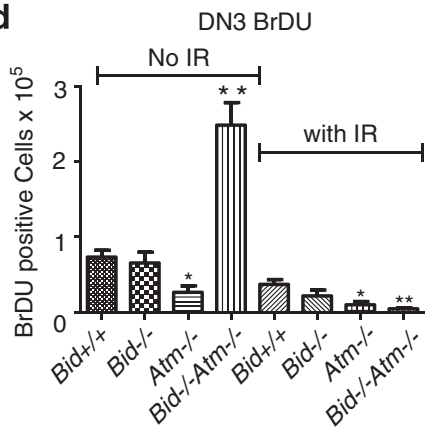

Figure 2 Bid - / - Atm - / - mice accumulate DN3 thymocytes. (a) Immunophenotype of double negative populations, DN1 (CD4 $\left.{ }^{-} \mathrm{CD} 8^{-} \mathrm{CD} 25^{-} \mathrm{CD} 44^{-}\right)$and DN3 $\left(\mathrm{CD} 4^{-} \mathrm{CD} 8{ }^{-} \mathrm{CD} 25^{+} \mathrm{CD}_{4} 4^{-}\right.$). Bid - I - Atm - I - mice accumulate DN3 cells. (b) DN3 cell number of untreated and IR-treated mice of the indicated genotypes. (c) BrdU incorporation of DN3 populations of untreated and IR-treated mice of the indicated genotypes. The BrdU-positive DN3 subsets cells were detected by intracellular staining with FITC-conjugated anti-BrdU antibody (BD Pharmingen) (d), quantification of data in (c). ${ }^{*} P<0.05$ and ${ }^{* *} P<0.01$ 
d). Importantly, this increased proliferation is noted in DN3 cells in vivo, consistent with a role for Bid in mediating the cell cycle checkpoint response to DNA damage generated during normal DN3 thymocyte development.

Atm - I - mice also display defective TCR rearrangement, reflected in loss of $\mathrm{CD} 3$ and TCR $\beta$ staining on double positive (DP) cells. In spite of the apparent increase in Bid - / - Atm - I - DN3 cells, Bid - / - Atm - / - DP cells do not display CD3 or TCR $\beta$ staining (Supplementary Figure S3). These results demonstrate that the increased number of DN3 cells in Bid-I-Atm - I- mice does not result in increased number of mature cells, due to persistent defects in differentiation in the absence of Atm.

Bid-I-Atm - I- bone marrow progenitors have normal numbers, normal replating efficiency, and normal response to IR. Recently, Puma was shown to inhibit T-cell leukemogenesis in an IR-induced leukemia model through regulation of bone marrow progenitor cell death. ${ }^{23}$ To determine if the increased leukemia latency of Bid-IAtm-I- mice was due to changes in bone marrow progenitor cells or their response to DNA damage, we evaluated the immunophenotype of HSC and progenitor cells (Supplementary Figure S4, panels A-F). There is no change in HSC-enriched lineage negative, Sca1 + Kit + (LSK) cells or progenitor (lineage negative, Sca1 $-\mathrm{Kit}+$ ) cells. To determine if the Bid-I-Atm-I- bone marrow progenitor cells display altered regenerative capacity, we performed serial methylcellulose replating assays. Fifty-thousand bone marrow cells of the indicated genotypes were plated in methylcellulose in the presence of IL-3, IL-6, SCF, and EPO as previously described. ${ }^{29}$ Colonies were counted and cells were replated on day 6 for a total of four platings. Bid-I - Atm - / - bone marrow demonstrates similar replating efficiency to Atm - Ibone marrow Supplementary Figure $S 4$ panel G). To determine if Bid-I-Atm-I- HSC and progenitor cells display an altered response to DNA damage, $\mathrm{Bid}+/+$, Bid-I-, Atm-I-, and Bid-I-Atm-I- mice were irradiated with $5 \mathrm{~Gy}$ IR. Mice were sacrificed after $48 \mathrm{~h}$, and immunophenotype analysis of HSC and progenitor populations was performed. There is no significant change in the response of Bid-I-Atm-I - HSC and progenitor populations to IR. The increased T-cell leukemia latency observed in Bid-I - Atm - I - mice cannot be accounted for by changes in HSC and progenitor populations, their regenerative capacity, or response to radiation.

Bid-I-Atm - I- DN3 thymocytes are more apoptotic. When challenged with IR, Bid-/-Atm-/- thymocytes display increased sensitivity to IR, and die significantly more than Bid - I - or Atm - I - thymocytes (Figure 1d). We further evaluated cell death in DN3 thymocytes by annexin $\mathrm{V}$ and activated caspase 3 staining. Bid-I-Atm-I- thymocytes display increased activated caspase 3 staining and annexin $\mathrm{V}$ staining at baseline (Figure 3). Interestingly, the percentage and number of activated caspase $3+B i d-I-A t m-I-$ DN3 cells appears to be disproportionately less than the annexin $\mathrm{V}+$ Bid-I - Atm - / - cells, suggesting the possibility that some of these cells may be undergoing caspase-independent cell death. Upon treatment with $5 \mathrm{~Gy} I \mathrm{R}$, Bid-/-Atm-/ - DN3 cells display increased percentage and number of activated caspase $3+$ and annexin $\mathrm{V}+$ cells relative to $\mathrm{Bid}+/+$, Bid-I-, or Atm-I- DN3 cells (Figure 3). Taken together, the above results are most consistent with a decreased ability of Bid-I - Atm - I - thymocytes to respond to DNA damage accrued during TCR $\beta$ recombination as well as to exogenous DNA damage. Of note, we do not observe similar accumulation of DN1 cells in Bid-/ - Atm-/ - mice. Furthermore, Bid-/ - Atm - / - DN1 cells do not accumulate or display increased apoptosis relative to $\mathrm{Atm}-/$ - DN1 cells (Supplementary Figure S5). Thus, the aberrant proliferation and apoptosis is restricted to the DN3 population, consistent with a role for Bid in response to DNA damage accrued during TCR $\beta$ recombination.

A prediction of the above model is that Bid-I-Atm - / DN3 cells should accumulate more DNA damage than $\mathrm{Bid}+/+$, Bid $-/-$, or Atm - / - thymocytes. To evaluate the integrity of DNA, we performed comet assays following DNA damage induced by IR or HU. Following IR, all genotypes displayed increased DNA damage. Notably, Bid-/Atm - / - thymocytes displayed significantly increased tail moment relative to all other genotypes following IR (Figures $4 \mathrm{a}$ and $b$ ), consistent with a role for Bid in mediating the DDR to IR in the absence of Atm. Following $\mathrm{HU}$, both Bid-/ - and Bid - / - Atm - / - thymocytes display increased tail moment relative to $\mathrm{Bid}+/+$ thymocytes (Figure $4 \mathrm{c}$ ). In addition, Bid-I-Atm - / - DN thymocytes display increased 53BP1 staining compared to Atm - / - DN thymocytes (Figure 4d) even without exogenous DNA damage. Taken together, the above results further support the model in which Bid facilitates an efficient DDR even in the absence of Atm. Of note, although we observe increased DNA damage by comet assay, ${ }^{30}$ we do not observe increased phospho- $\gamma-\mathrm{H} 2 \mathrm{~A}$.X staining in either Atm-I - or Bid-I - Atm - I - thymocytes (Supplementary Figure S6). This is predicted, as phosphorylation of $\gamma-\mathrm{H} 2 \mathrm{~A} . \mathrm{X}$ is dependent on functional Atm.

$A$ recent report demonstrated increased reactive oxygen species (ROS) in mice harboring Bid that is mutated at Atm/ Atr phosphorylation sites. ${ }^{22}$ We asked whether the accumulation of DNA damage in Bid-I - Atm - / - thymocytes could be attributed to increased ROS in the absence of Bid. We find no difference between the ROS produced in Atm - / - and Bid-I-Atm-I - thymocytes. Furthermore, there is no difference in DCFDA fluorescence in DN1 or DP thymocytes (Supplementary Figure S7). Although we cannot strictly rule out a role for ROS, our data are inconsistent with a dominant role for increased ROS in causing the increased DNA damage observed in Bid-I-Atm-I - thymocytes. Further studies will require more sensitive and specific methods to measure ROS in these cells.

Loss of Bid has no effect on the cell death response to ionization radiation in DP thymocytes. Our data suggest that Bid has a survival role in vivo in early thymocytes' development in developmental stages where DNA damage occurs. In thymocytes development, TCR $\beta$ chains are rearranged in the DN3 stage and $\mathrm{TCR} \alpha$ chains are rearranged in the DP stage. To determine whether the cell death effects noted in Bid-/-Atm-I- DN3 cells were also observed in the DP thymocyte population, we evaluated cell 

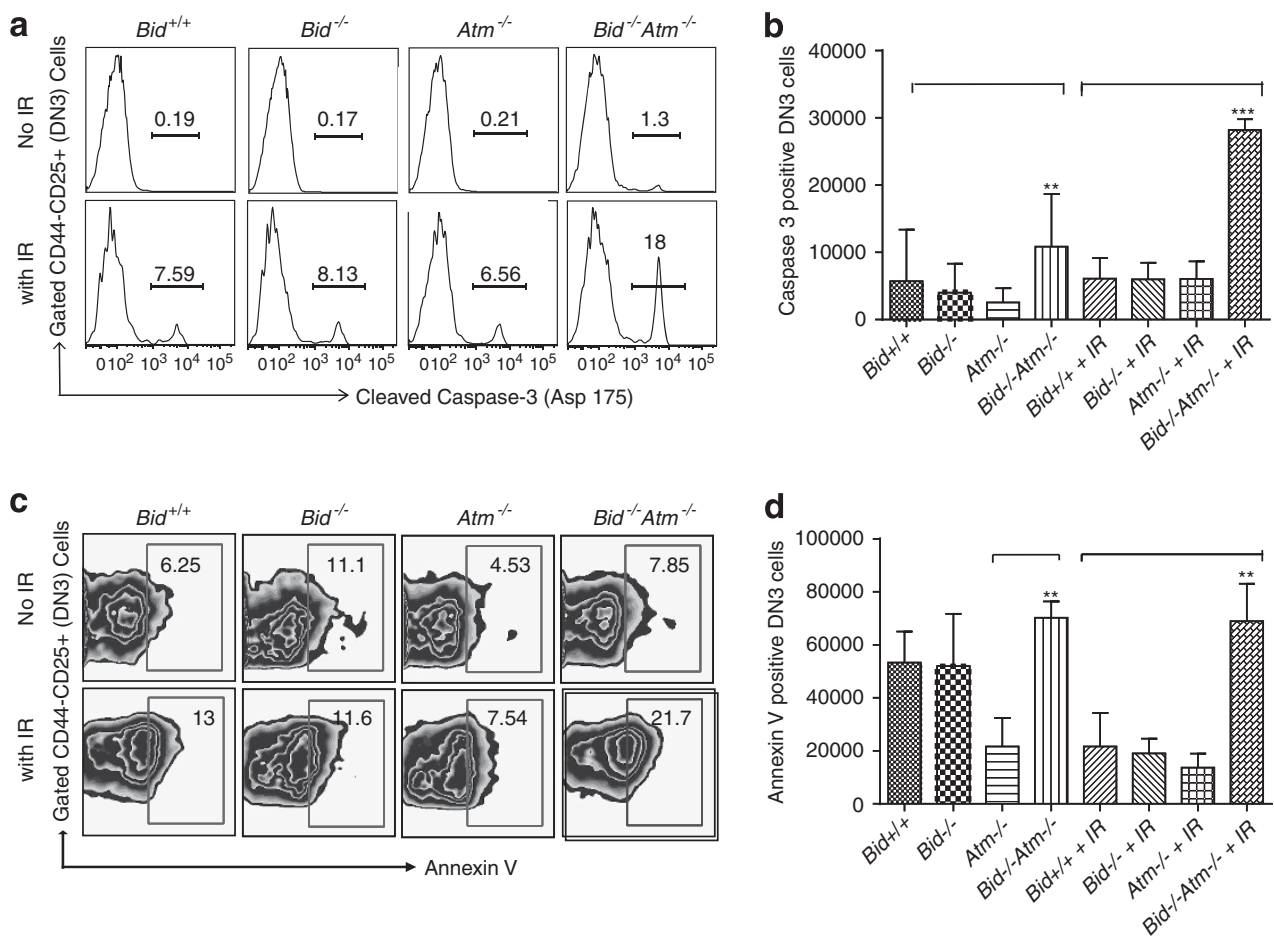

Figure 3 Bid - / - Atm - / - DN3 cells are more sensitive to IR. (a) Relative expression of intracellular activated caspase-3 in DN3 cells from Bid + /+, Bid - / - , $A t m-/-$, and Bid - / - Atm - / - mice by flow cytometric analysis. Mice of the indicated genotypes were treated with 5 Gy IR and thymocytes were harvested after $12 \mathrm{~h}$. After surface staining with anti-CD4, anti-CD8 $\alpha$, anti-CD44, and anti-Cd25, thymocytes were fixed, permeabilized, and stained with anti-cleaved Caspase-3 (Cell Signaling) followed by staining with Alexa Fluor 488-conjugated anti-rabbit secondary antibody. (b) Caspase-3-positive subsets of DN3 cells were measured by flow cytometry and quantitative analysis was performed. Data are from three sets of mice. (c) Flow cytometry analysis of apoptosis of the DN3 population by annexin V staining. (d) Quantification of apoptosis by annexin $V$ staining of DN3 subsets from the indicated genotypes of mice. ${ }^{*} P<0.05,{ }^{* \star} P<0.01$ and ${ }^{* \star *} P<0.001$

death of DP thymocytes both at baseline and following IR. Both Atm-I- and Bid-I-Atm-I- DP thymocytes display protection from death after IR (Figure 5) relative to Bid $+/+$ and $\mathrm{Bid}-\mathrm{I}$ - thymocytes, consistent with reported results for Atm - / - thymocytes. ${ }^{31,32}$ Importantly, there is no difference between Bid $+/+$ and Bid-I- DP thymocytes, or between Atm-I - or Bid-I-Atm-I - DP thymocytes with respect to percentage or number of DP cells (Figures $5 a$ and $b$ ), or annexin $\mathrm{V}+\mathrm{DP}$ cells (Figures $5 \mathrm{c}$ and $\mathrm{d}$ ) before or after IR. This is distinct from DN3 thymocytes in which Bid - / - Atm - / - DN3 thymocytes accumulate at baseline, but are more sensitive to DNA damage-induced cell death (Figure3).

Bid - I - and Bid - I-Atm - I - DN3 thymocytes display decreased pChk1 accumulation following IR or HU. We have previously shown that Bid-/- MPC cells display a defective Atr-mediated DDR, with decreased accumulation of ATRIP at nuclear foci, and decreased activation of Chk1 by phosphorylation following agents inducing replicative stress, such as $\mathrm{HU} .{ }^{21}$ Our current results suggest that Bid mediates an Atm-independent DDR. Atr directs the DDR to replicative stress, and the Atr-mediated DDR is upregulated in Atm - / - cells to compensate for loss of the Atm-mediated DDR. ${ }^{12}$ To evaluate the Atr-mediated DDR in the absence of Atm, we utilized an intracellular phospho-Chk1 flow assay to monitor Chk1 phosphorylation on a per-cell basis in DN3 cells. The percentage of $\mathrm{Bid}-/-$ and $\mathrm{Bid}-/-\mathrm{Atm}-/-$ DN3 thymocytes that display phospho-Chk1 positivity following both
IR and HU (Figure 6) is decreased. These results are consistent with a defective Atr-mediated DDR in the absence of Bid.

\section{Discussion}

Several independent groups have shown that Bid has a role in the DDR that is independent of its apoptotic function, but dependent on phosphorylation by Atm and/or Atr. $^{17,18}$ In addition, we have demonstrated a role for Bid in the DDR to replicative stress mediated by Atr. ${ }^{19,21,33}$ To probe Atmindependent roles for Bid in vivo, we have crossed Bid - / mice to Atm - / - mice. Surprisingly, we found that loss of Bid increases the latency of leukemogenesis in Atm - / - mice. We find that Bid - I - Atm - I - thymi accumulate DN3 cells, the stage at which TCR $\beta$ chain rearrangement occurs. These Bid - I - Atm - I - DN3 cells display evidence of a defective DDR, with increased proliferation, decreased Chk1 phosphorylation (loss of checkpoint control), increased tail moment by comet assay, increased 53BP1 foci formation (increased DNA damage), and increased sensitivity to DNA damage induced by IR or $\mathrm{HU}$. These results are consistent with an Atmindependent role for Bid in mediating a proper DDR. We propose a model in which the delay in leukemogenesis is due to loss of cells that accumulate DNA damage at a higher rate in Bid-/ - Atm-/ - mice, increasing the latency for accumulation of leukemogenic mutations (Figure 7).

Interestingly, mice deficient in Bid and the pre-TCR also display accumulation of DN3 cells, but no corresponding 
a
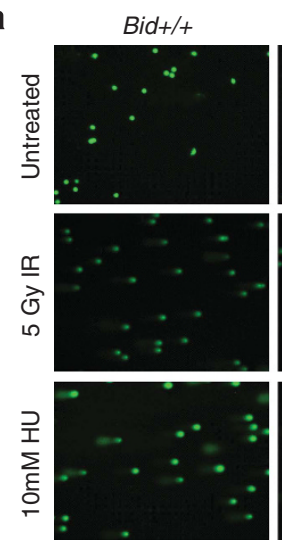

b

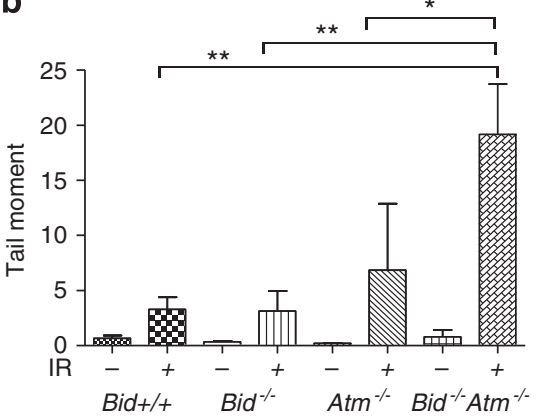

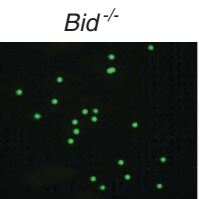
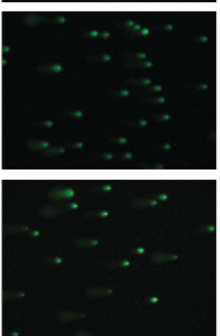
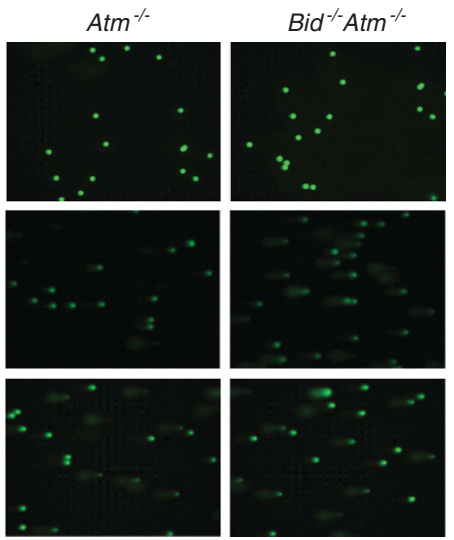

C

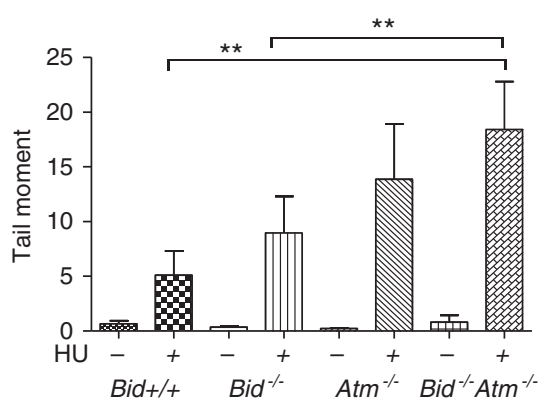

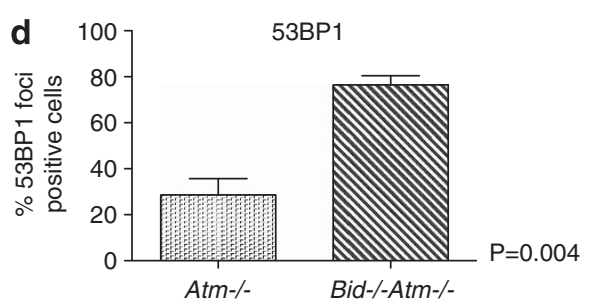

Figure 4 Bid - / - Atm - / - thymocytes display significantly increased DNA damage after IR and HU and Bid - / - Atm - / - DN thymocytes display increased DNA damage at baseline. Micrographs showing comet assays of thymocytes from the indicated genotypes treated with 5 Gy IR or $10 \mathrm{mM} \mathrm{HU}$, and harvested after $24 \mathrm{~h}$. The untreated and treated cells were collected in ice-cold PBS and an alkaline comet assay (Trevigen) was performed. The samples were run in alkaline electrophoresis solution at $21 \mathrm{~V}$ for $30 \mathrm{~min}$. After staining with SYBR Green I, samples were examined using a Leica DM IRBE inverted wild-field microscope. (b, c) At least 75 randomly chosen cells per sample without or with (b) IR and, (c) HU treatment were analyzed using CometScore Program version 1.5. Atm - / - and Bid - / - Atm - / - DN thymocytes were isolated by magnetic bead sorting and stained with anti-53BP1. The percentage of cells with at least five 53BP1 positive nuclear foci was determined. At least 75 randomly chosen cells were evaluated per sample. ${ }^{*} P<0.05$ and ${ }^{* *} P<0.01$

increase in more mature DP cells. ${ }^{27}$ In this setting, accumulation of DN3 cells was attributed to protection from a p53mediated cell death signal based on in vitro cell death assays, and measurement of annexin $\mathrm{V}$ positivity of all cells in the thymus. In Bid-/-Atm-I- mice, we see a similar accumulation of DN3 cells. However, we do not see protection from cell death, but rather see that caspase 3 activity, and BrdU incorporation are increased in Bid - / - Atm - / - DN3 cells, consistent with the accumulation of DN3 cells due to increased proliferation. In the setting of a pre-TCR - /mouse model, cell death is initiated by loss of a pre-TCRmediated survival signal. In the setting of Atm - / - thymocytes, cell death is initiated by excessive DNA damage due to loss of DNA repair. It is notable that experiments to evaluate the reconstitution ability of $\mathrm{Bid}-/$ - cells also demonstrated an increase in DN3 cells. ${ }^{28}$ These studies also demonstrated increase in pro-B cells, the B-cell precursor in which a DDR is activated upon immunoglobulin gene rearrangement. Loss of
Bid thus clearly influences the control of multiple populations of cells in which DNA damage control is critical.

Of note, we do not see increased proliferation or increased caspase activation in any other Bid-/ - Atm - / - thymocyte population, including the DP population in which $\mathrm{TCR} \alpha \beta$ chains undergo recombination. One notable difference between DP and DN3 cells is proliferation. DN3 cells are rapidly proliferating due to signaling through the pre-TCR, whereas DP cells are not. The increased role for Bid in the setting of DNA damage in replicating cells is consistent with a role for Bid in the Atr-mediated DDR.

$A$ recent paper implicates $\mathrm{Bid}$ in regulation of $\mathrm{ROS}$ downstream of Atm. ${ }^{22}$ Interestingly, treatment of Atm - / mice with the antioxidant $\mathrm{N}$-acetyl cysteine also decreases the incidence of leukemogenesis in Atm-/- mice, ${ }^{34}$ suggesting that regulation of ROS by Atm may have a role in leukemogenesis. We do not find increased ROS in Bid-I-Atm-I - DN3 cells, therefore we cannot explain 

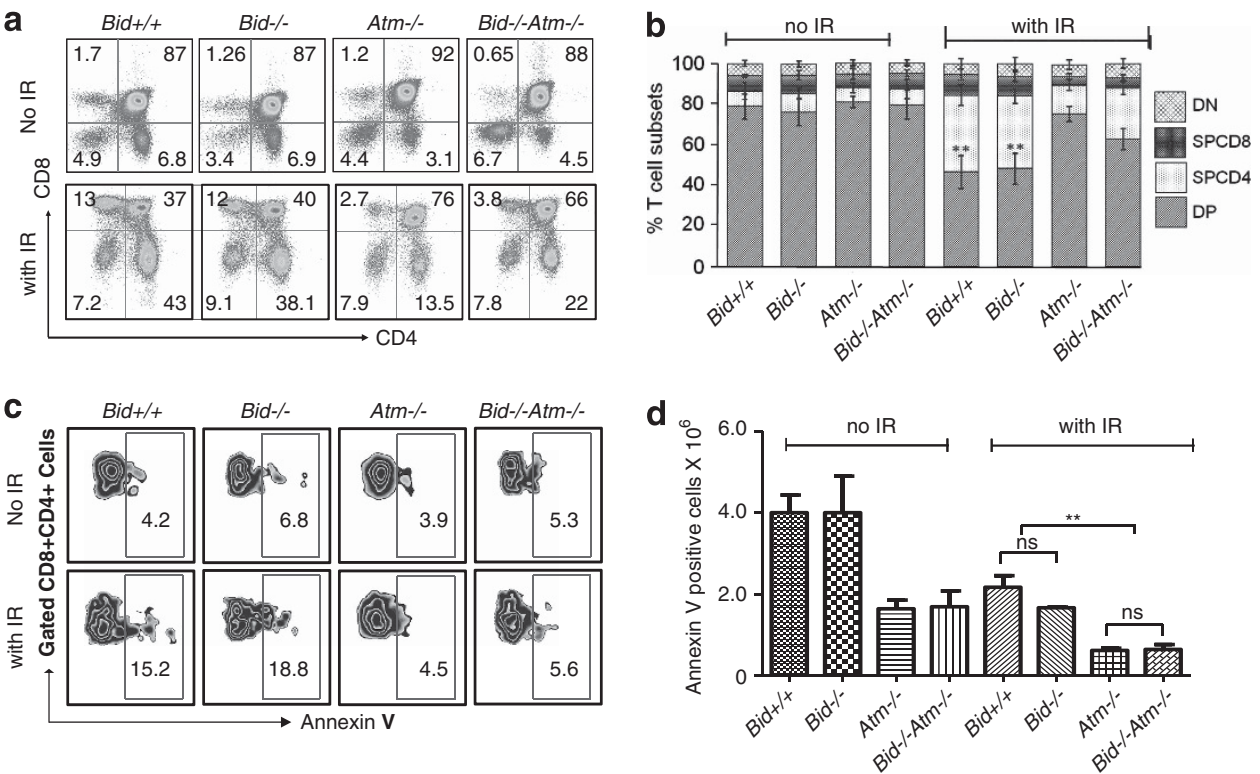

Figure 5 Both Atm - / - and Bid - / - Atm - / - DP populations are more resistant to IR. (a) Flow cytometry analysis of T-cell subsets from thymocytes of mice untreated and irradiated with $5 \mathrm{~Gy}$ IR harvested after $48 \mathrm{~h}$. After IR treatment, the Atm - I - and Bid - / - Atm - / - DP populations are protected from cell death. (b) Three sets of irradiated and untreated mice of each genotype were analyzed and the percentage of the indicated thymocyte subsets are presented. Error bars denote S.D. (c) Flow cytometry analysis of apoptosis of the DP population by annexin V staining. (d) Quantification of the data in (c). ${ }^{* *} P<0.01$
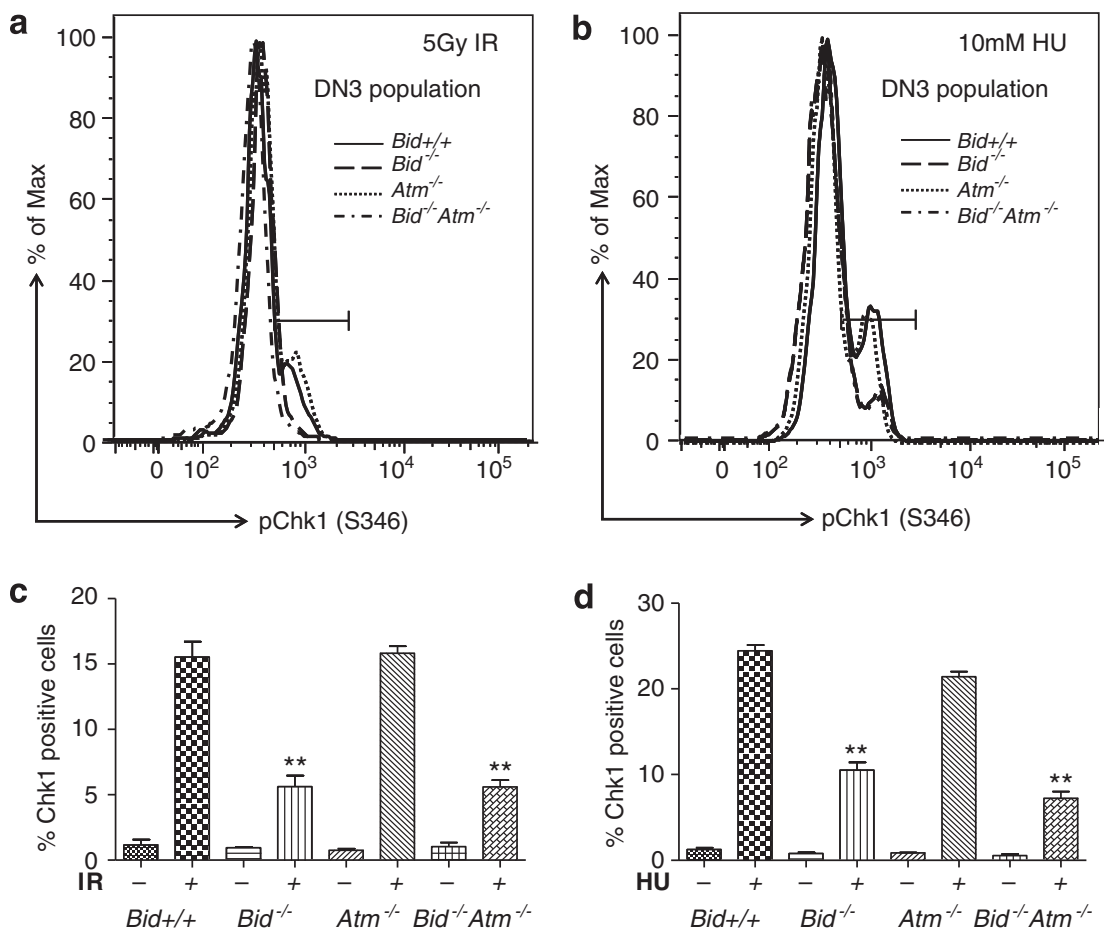

Figure 6 Bid - / - and Bid - / - Atm - / - DN3 thymocytes display decreased Chk1 phosphorylation following IR and HU. (a, b) Total thymocytes were treated with (a) 5 Gy IR and harvested after $4 \mathrm{~h}$ or, (b) $10 \mathrm{mM}$ hydroxyurea and harvested after $2 \mathrm{~h}$. Cells were stained with anti-CD4, anti-CD8, anti-CD25, and anti-CD44 for 30 min. Chk1 phosphorylation was detected by intracellular staining with anti-pChk1 (S345) antibody (Cell signaling, no. 2348) and Alexa Fluor 488-conjugated Goat anti-Rabbit lgG antibody (Invitrogen). (c) Quantitative analysis of data in (a), showing the percentage of pChk1-positive DN3 subsets from three independent experiments. (d) Quantitative analysis of data in (b), showing the percentage of pChk1-positive DN3 subsets from three independent experiments. ${ }^{* *} P<0.01$

the increased latency of leukemogenesis or the increased DNA damage by increased ROS. However, we do not rule out a role for Bid in ROS regulation and Atm-mediated leukemogenesis. Of note, loss of Bid may not have the same effect as loss of phosphoregulation of Bid. ${ }^{22}$ Indeed, accumulating evidence would suggest that phosphorylation might alter the biological function of Bid to a survival role, perhaps by inhibiting caspase cleavage and mitochondrial localization. 
T Lymphocyte
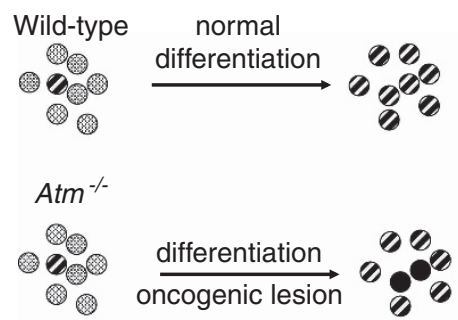

$\mathrm{Bid}^{-/-} \mathrm{Atm}^{-/-}$
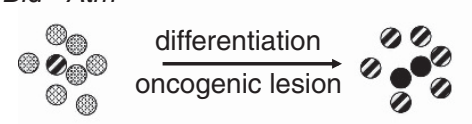

normal
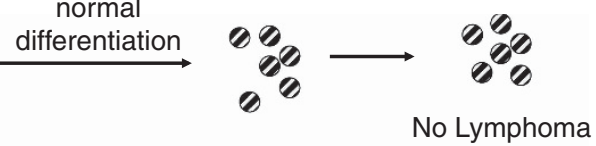

No Lymphoma
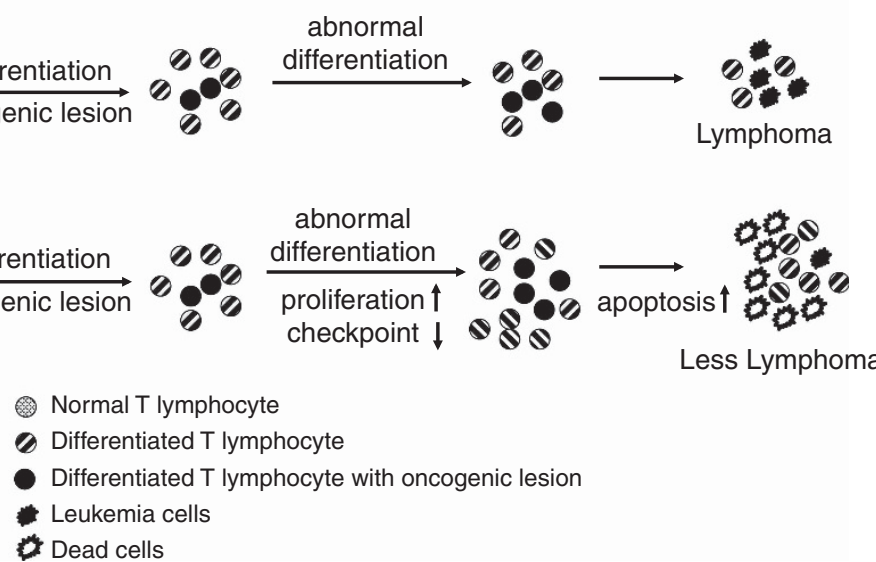

Less Lymphoma

Figure 7 Model for the role of Bid in T-cell lymphoblastic leukemia/lymphoma. Atm - / - mice develop T-cell lymphoblastic leukemia/lymphoma within 3 months, due to an aberrant DNA damage response to DNA strand breaks accrued during T-cell development. Bid - / - Atm - / - mice display increased proliferation and cell death in the DN3 thymocyte progenitor population, and increased latency of tumorigenesis. We propose a model in which loss of Bid further impairs the DNA damage response in Atm - I - thymocytes, and increases the DNA damage and cell death of a vulnerable population of cells, increasing tumor latency

Our results, however, are most consistent with a role for Bid in mediating an Atm-independent DDR, facilitating proper checkpoint control to maintain genomic integrity.

Distinct from the IR-induced model of T-cell lymphomagenesis, Atm $-/-$ mice have an impaired DDR required for normal T-cell differentiation due to DNA strand breaks that are accrued during TCR recombination. Loss of Atm thus results in leukemogenesis, but the transformed cell is a thymic progenitor, as loss of Rag recombination abrogates leukemogenesis in Atm-/ - mice. We find that loss of Bid in addition to Atm further impairs the DDR in DN3 thymocytes. The end effect on leukemogenesis is to delay the onset of leukemia by removing cells with potentially oncogenic lesions (Figure 7). Therefore, in the hematopoietic system, the balance between life and death is carefully regulated and modified in a cell-type-specific manner to respond to specific biological environments. The BH3-only members of the Bcl-2 family have important roles in the regulation of cell death, and the final outcome of their influence is determined in a cell-typeand context-specific manner.

\section{Materials and methods}

Mice. All experiments with mice conformed to the guidelines of the Vanderbilt University Medical Center Animal Ethics committee. Bid +/+, Bid - / - C57/ $\mathrm{BL6}^{35^{17}}$ and Atm- / $\left(129 \mathrm{~S} / \mathrm{SvEvTac}-\right.$ Atm $^{\text {tm1 Awb }}$, Jackson Laboratory, Bar Harbor, ME, USA ${ }^{26}$ (6-8 weeks old) were maintained in the Preston Research Building at Vanderbilt Medical Center. The Bid $-I-A t m-I-$ mice were generated by intercrossing of the Bid $-/$ - and Atm $-/$ - parental strains. Mice were monitored for 180 days for signs of illness and time of tumor onset.

BrdU incorporation. To determine the proliferation status of cells, Bid $+/$ ,+ Bid $-I-, A t m-I-$, and Bid - I-Atm - I - mice were injected with BrdU (3.3 mg per mouse injected intraperitoneally). Four hours post injection, the mice were sacrificed and thymocytes were analyzed for BrdU incorporation (BrdU Flow Kit, BD Biosciences, San Jose, CA, USA), according to the manufacturer's instructions. Briefly, $1 \times 10^{6}$ cells were stained for $30 \mathrm{~min}$ with surface antibody specific for $\mathrm{CD} 4, \mathrm{CD} 8, \mathrm{CD} 44$, and $\mathrm{CD} 25$ to identify progenitor populations. Then cells were fixed and permeabilized with BD Cytofix/Cytoperm Buffer, and incubated with BD Cytoperm Plus Buffer followed by an additional short fixation with BD Cytofix/Cytoperm Buffer. The incorporated BrdU was exposed by treatment with $30 \mu \mathrm{g}$ DNase for $1 \mathrm{~h}$ at $37^{\circ} \mathrm{C}$ and probed with FITC-conjugated anti-BrdU antibody (BD Biosciences) for $20 \mathrm{~min}$ at room temperature.

Apoptosis analysis. To measure apoptosis, thymocytes from Bid $+/+$, Bid - I-, Atm - I-, and Bid - I-Atm - I- mice were either left untreated or subjected to $5 \mathrm{~Gy}$ IR or HU. Cells were stained with the appropriate antibodies to identify progenitor populations and annexin V (BD Bioscience) and analyzed by flow cytometry. For in vitro death assays, thymocytes from Bid $+/+$, Bid $-/-$, Atm $-/-$, and Bid $-/-A t m-I-$ mice were suspended in RPMl 10 media either with or without $1 \mathrm{mM} \mathrm{HU}$ or directly treated with $5 \mathrm{~Gy} I R$. Cells were collected at the indicated time points and stained with annexin V-FITC and analyzed by flow cytometry.

Immunofluorescent staining and FACS analysis. Immunophenotype analysis of thymocyte populations was performed as described in ${ }^{36}$ using monoclonal antibodies specific to thymocyte cell populations. Stained cells were analyzed on an LSRII (Becton Dickinson, BD biosciences) flow cytometer.

DNA damage analysis by comet assay. The thymocyte cells from Bid $+/+$, Bid $-/-, A t m-/-$, and Bid - / - Atm - / - mice were treated with $10 \mathrm{mM} \mathrm{HU}$ and $5 \mathrm{~Gy}$ ionization radiation, and incubated overnight. The untreated and treated cells were collected in ice-cold PBS and the alkaline comet assay was performed using the CometAssay Kit (Trevigen, Gaithersburg, MD, USA) according to the manufacturer's instructions. Briefly, cells were mixed with molten LM Agarose and pipetted onto a CometSlide. After incubation with Lysis Solution and Alkaline Solution, alkaline gel electrophoresis was performed at $22 \mathrm{~V}$ for $10 \mathrm{~min}$. After incubation with $70 \%$ ethanol for $5 \mathrm{~min}$, the slides were stained with SYBR Green I. Then, samples were examined using a Leica DM IRBE inverted wildfield microscope and analyzed by CometScore Program version 1.5 (Trevigen, Gaithersburg, MD, USA).

Assessment of DNA damage and cell death by intracellular phospo- $\gamma$ H2A.X levels, and intracellular cleaved caspase-3 levels, respectively. To measure DNA damage, Bid $+/+$, Bid $-/-$, 
Atm $-I-$, and Bid $-I-A t m-I-$ mice were treated with 5 Gy ionization radiation for $4 \mathrm{~h}$ to measure phospo- $\gamma$ H2A.X, or $12 \mathrm{~h}$ to measure cleaved caspase 3 levels. Mice were sacrificed and $1 \times 10^{6}$ thymocytes were stained for $30 \mathrm{~min}$ with surface antibody specific for $C D 4, C D 8, C D 44$, and $C D 25$ to identify progenitor populations. For intracellular staining of phospho- $\gamma \mathrm{H} 2 \mathrm{~A} . \mathrm{X}$ or cleaved caspase-3, thymocytes were fixed and permeabilized with BD Cytofix/Cytoperm Buffer, and incubated with BD Cytoperm Plus Buffer followed by an additional short fixation with BD Cytofix/Cytoperm Buffer. Then, cells were stained with either antiphospho-H2A.X (Upstate, NY, USA no. 07-164) or cleaved caspase-3 (Asp175) antibody (dilution 1:800) (Cell signaling technologies, MA, USA, no. 9661) and then Alexa Fluor 488-conjugated Goat anti-Rabbit IgG antibody (Invitrogen, Life Technologies, Grand Island, NY, USA) as a secondary antibody. The phospho- $\gamma$ H2A.X or cleaved caspase- 3 expression of thymic progenitors gated by surface markers was measured by flow cytometry. The data were analyzed using Flow Jo software version 9.3.3 (Treestar, Inc., Ashland, OR, USA).

53BP1 staining. Total thymocytes were stained with biotin-conjugated antiCD4 and CD8, followed by sheep anti-rat-conjugated Dyna-beads. DN cells were isolated by removing $\mathrm{CD}^{+}$and $\mathrm{CD} 8^{+}$cells using a magnet. Cells were immobilized on slides by cytospin. Cells were fixed in $4 \%$ paraformaldehyde and permeabilized with $0.5 \%$ triton $\mathrm{X}-100$ solution. Cells were visualized and foci were counted on a Leica DM IRBE inverted wildfield microscope.

Measurement of phospho-Chk1. Cells were stained with anti-pChk1 (S345) antibody (Cell signaling, no. 2348) as above, and Alexa Fluor 488-conjugated Goat anti-Rabbit IgG antibody (Invitrogen), and analyzed by flow cytometry.

Measurement of ROS levels. To measure ROS levels, bone marrow and thymocyte stains were modified to make the FL- 1 channel available for $\mathrm{H}_{2}$ DCFDA staining (2' $7^{\prime}$-dichlorofluorescencein diacetate, molecular probes). $\mathrm{H}_{2}$ DCFDA is a nonpolar compound that readily diffuses into cells, where it is cleaved by intracellular esterases to form $\mathrm{H}_{2} \mathrm{DCF}$, and thereby is trapped inside the cells. $\mathrm{H}_{2} \mathrm{DCF}$ is oxidized to the highly fluorescent 2,7-dichlorofluorescein (DCF) following ROS generation. Antibodies used were: APC-cy7-conjugated anti-CD4 and -CD8, PE-conjugated CD44, PerCP Cy5.5-conjugated CD25, and APC-conjugated C-kit. After antibody staining, cells were incubated with $5 \mu \mathrm{M}$ DCFDA for $15 \mathrm{~min}$ at $37^{\circ} \mathrm{C}$ followed by flow cytometry. The oxidized fluorescent DCF following ROS generation emission at $488 \mathrm{~nm}$ was measured on a FACS Calibur flow cytometer (Becton Dickenson, LSRII, BD Biosciences) and analyzed using the Flow Jo analysis software as above.

Statistical analysis. Prism Software (GraphPad Software Inc., La Jolla, CA, USA) was used for generating Kaplan-Meier animal survival plots and for performing statistical analysis (using a rank test) of mouse survival. All other statistical analysis used a $t$ (one-tailed, assuming equal variances or two-tailed, assuming unequal variance). $P$-values $<0.05$ were considered to indicate statistical significance.

\section{Conflict of Interest}

The authors declare no conflict of interest.

Acknowledgements. We thank Dr. Jennifer Pietenpol, Dr. Scott Hiebert, Dr. David Cortez, Dr. Christine Eischen, and Dr. Elizabeth Yang, for many helpful discussions. This work was supported by funds from the Sidney Kimmel Foundation, the G and P Foundation, ACS IRG-58-009-47, NIH K08 CA098394, and R01 HL088347 to SSZ, and the Lady Tata Memorial Trust to SB. Cell imaging experiments were performed in the VUMC Cell Imaging Shared Resource.

1. Soria G, Polo SE, Almouzni G. Prime, repair, restore: the active role of chromatin in the DNA damage response. Mol Cell 2012; 46: 722-734.

2. Roos WP, Kaina B. DNA damage-induced apoptosis: From specific DNA lesions to the DNA damage response and apoptosis. Cancer Lett 2012; doi:10.1016/j.canlet.2012.01.007.

3. Shiloh Y. ATM and related protein kinases: safeguarding genome integrity. Nat Rev Cancer 2003; 3: 155-168.
4. Bartek J, Lukas C, Lukas J. Checking on DNA damage in S phase. Nat Rev Mol Cell Biol 2004; 5: 792-804.

5. Ciccia A, Elledge SJ. The DNA damage response: making it safe to play with knives. $\mathrm{Mol}$ Cell 2010; 40: 179-204.

6. Lovejoy CA, Cortez D. Common mechanisms of PIKK regulation. DNA Repair 2009; 8 : 1004-1008.

7. Chen BP, Li M, Asaithamby A. New insights into the roles of ATM and DNA-PKcs in the cellular response to oxidative stress. Cancer Lett 2011; 327: 103-110.

8. Cimprich KA, Cortez D. ATR: an essential regulator of genome integrity. Nat Rev Mol Cell Biol 2008; 9: 616-627.

9. Burrows AE, Elledge SJ. How ATR turns on: TopBP1 goes on ATRIP with ATR. Genes Dev 2008; 22: 1416-1421.

10. Lopez-Contreras AJ, Fernandez-Capetillo O. The ATR barrier to replication-born DNA damage. DNA Repair 2010; 9: 1249-1255.

11. Smith J, Tho LM, Xu N, Gillespie DA. The ATM-Chk2 and ATR-Chk1 pathways in DNA damage signaling and cancer. Adv Cancer Res 2010; 108: 73-112.

12. Brown EJ, Baltimore D. Essential and dispensable roles of ATR in cell cycle arrest and genome maintenance. Genes Dev 2003; 17: 615-628.

13. Prives C. Signaling to p53: breaking the MDM2-p53 circuit. Cell 1998; 95: 5-8.

14. Jeffers JR, Parganas E, Lee $Y$, Yang $C$, Wang J, Brennan $J$ et al. Puma is an essential mediator of p53-dependent and -independent apoptotic pathways. Cancer Cell 2003; 4: 321-328.

15. Kim H, Tu HC, Ren D, Takeuchi O, Jeffers JR, Zambetti GP et al. Stepwise activation of BAX and BAK by tBID, BIM, and PUMA initiates mitochondrial apoptosis. Mol Cell 2009; 36: 487-499.

16. Zinkel SS, Yin XM, Gross A. Rejuvenating Bi(d)ology. Oncogene 2012; doi:10.1038/ onc. 2012.454

17. Zinkel SS, Hurov KE, Ong C, Abtahi FM, Gross A, Korsmeyer SJ et al. A role for proapoptotic BID in the DNA-damage response. Cell 2005; 122: 579-591.

18. Kamer I, Sarig R, Zaltsman Y, Niv H, Oberkovitz G, Regev L et al. Proapoptotic BID is an ATM effector in the DNA-damage response. Cell 2005; 122: 593-603.

19. Liu Y, Vaithiyalingam S, Shi Q, Chazin WJ, Zinkel SS. BID binds to replication protein $A$ and stimulates ATR function following replicative stress. Mol Cell Biol 2011; 31: 4298-4309.

20. Liu Y, Aiello A, Zinkel SS. Bid protects the mouse hematopoietic system following hydroxyurea-induced replicative stress. Cell Death Differ 2012; 19: 1602-1612.

21. Liu Y, Bertram CC, Shi Q, Zinkel SS. Proapoptotic Bid mediates the Atr-directed DNA damage response to replicative stress. Cell Death Differ 2011; 18: 841-852.

22. Maryanovich M, Oberkovitz G, Niv H, Vorobiyov L, Zaltsman Y, Brenner $O$ et al. The ATMBID pathway regulates quiescence and survival of haematopoietic stem cells. Nat Cell Biol 2012; 14: 535-541.

23. Michalak EM, Vandenberg CJ, Delbridge AR, Wu L, Scott CL, Adams JM et al. Apoptosispromoted tumorigenesis: gamma-irradiation-induced thymic lymphomagenesis requires Puma-driven leukocyte death. Genes Dev 2010; 24: 1608-1613.

24. Labi V, Erlacher M, Krumschnabel G, Manzl C, Tzankov A, Pinon J et al. Apoptosis of leukocytes triggered by acute DNA damage promotes lymphoma formation. Genes Dev 2010; 24: 1602-1607.

25. Liao MJ, Van Dyke T. Critical role for Atm in suppressing $V(D) J$ recombination-driven thymic lymphoma. Genes Dev 1999; 13: 1246-1250.

26. Barlow C, Hirotsune S, Paylor R, Liyanage M, Eckhaus M, Collins F et al. Atm-deficient mice: a paradigm of ataxia telangiectasia. Cell 1996; 86: 159-171.

27. Mandal M, Crusio KM, Meng F, Liu S, Kinsella M, Clark MR et al. Regulation of lymphocyte progenitor survival by the proapoptotic activities of Bim and Bid. Proc Natl Acad Sci USA 2008; 105: 20840-20845.

28. Shen $\mathrm{H}, \mathrm{Yu} \mathrm{H}$, Liang PH, Xufeng R, Song $\mathrm{Y}, \mathrm{HuX}$ et al. Bid is a positive regulator for donorderived lymphoid cell regeneration in gamma-irradiated recipients. Exp Hematol 2011; 39: 947-957; e941.

29. Zinkel SS, Ong CC, Ferguson DO, Iwasaki H, Akashi K, Bronson RT et al. Proapoptotic BID is required for myeloid homeostasis and tumor suppression. Genes Dev 2003; 17: 229-239.

30. Burlinson B. The in vitro and in vivo comet assays. Methods Mol Biol 2012; 817: 143-163.

31. Xu Y, Ashley T, Brainerd EE, Bronson RT, Meyn MS, Baltimore D et al. Targeted disruption of ATM leads to growth retardation, chromosomal fragmentation during meiosis, immune defects, and thymic lymphoma. Genes Dev 1996; 10: 2411-2422.

32. Xu Y, Baltimore D. Dual roles of ATM in the cellular response to radiation and in cell growth control. Genes Dev 1996; 10: 2401-2410.

33. Liu Y, Aiello A, Zinkel SS. Bid protects the mouse hematopoietic system following hydroxyurea-induced replicative stress. Cell Death Differ 2012; 19: 1602-1612.

34. Reliene R, Schiestl RH. Antioxidant N-acetyl cysteine reduces incidence and multiplicity of lymphoma in Atm deficient mice. DNA Repair 2006; 5: 852-859.

35. Liu Y, Bertram CC, Shi Q, Zinkel SS. Proapoptotic Bid mediates the Atr-directed DNA damage response to replicative stress. Cell Death Differ 2011; 18: 841-852.

36. Biswas S, Shi Q, Matise L, Cleveland S, Dave U, Zinkel S. A role for proapoptotic Bax and Bak in T-cell differentiation and transformation. Blood 2010; 116: 5237-5246. 